\title{
High-Affinity Binding of the NC1 Domain of Collagen VII to Laminin 5 and Collagen IV
}

\author{
Raymond Brittingham \\ Thomas Jefferson University \\ Jouni Uitto \\ Thomas Jefferson University \\ Andrzej Fertala \\ Thomas Jefferson University
}

Follow this and additional works at: https://jdc.jefferson.edu/dcbfp

Part of the Medical Biochemistry Commons

\section{Let us know how access to this document benefits you}

\section{Recommended Citation}

Brittingham, Raymond; Uitto, Jouni; and Fertala, Andrzej, "High-Affinity Binding of the NC1 Domain of Collagen VII to Laminin 5 and Collagen IV" (2006). Department of Dermatology and Cutaneous Biology Faculty Papers. Paper 2.

https://jdc.jefferson.edu/dcbfp/2

This Article is brought to you for free and open access by the Jefferson Digital Commons. The Jefferson Digital Commons is a service of Thomas Jefferson University's Center for Teaching and Learning (CTL). The Commons is a showcase for Jefferson books and journals, peer-reviewed scholarly publications, unique historical collections from the University archives, and teaching tools. The Jefferson Digital Commons allows researchers and interested readers anywhere in the world to learn about and keep up to date with Jefferson scholarship. This article has been accepted for inclusion in Department of Dermatology and Cutaneous Biology Faculty Papers by an authorized administrator of the Jefferson Digital Commons. For more information, please contact: JeffersonDigitalCommons@jefferson.edu. 


\title{
High-Affinity Binding of the NC1 Domain of Collagen VII to Laminin 5 and Collagen IV
}

\author{
Raymond Brittingham ${ }^{a}$, Jouni Uitto ${ }^{a}$, Andrzej Fertala $^{a^{*}}$
}

${ }^{\mathrm{a}}$ Department of Dermatology and Cutaneous Biology, Jefferson Medical College, and Jefferson Institute of Molecular Medicine, Thomas Jefferson University, Philadelphia, Pennsylvania, 19107, U.S.A.

*Correspondence to Andrzej Fertala, Department of Dermatology and Cutaneous Biology, Jefferson Medical College, Thomas Jefferson University, BLSB, Room 424, 233 S. 10th Street, Philadelphia, PA 19107, U.S.A (corresponding address is the same as that in the cover letter).

Tel: $215-503-0113$

Fax: 215-503-5788

E-mail: andrzej.fertala@jefferson.edu

Keywords: Extracellular matrix of connective tissue; collagen VII; collagen IV; laminin 5; collagen mutations; biosensor; epidermolysis bullosa 


\begin{abstract}
Anchoring functions of collagen VII depend on its ability to form homotypic fibrils and to bind to other macromolecules to form heterotypic complexes. Biosensor-based binding assays were employed to analyze the kinetics of the $\mathrm{NC1}$ domain-mediated binding of collagen VII to laminin 5, collagen IV, and collagen I. We showed that collagen VII interacts with laminin 5 and collagen IV with $\mathrm{K}_{d}$ values of $10^{-9} \mathrm{M}$. In contrast, the NC1-mediated binding to collagen I was weak with a $\mathrm{K}_{d}$ value of $10^{-6} \mathrm{M}$. Binding assays also showed that the NC1 domain utilizes the same region to bind to both laminin 5 and collagen IV.

We postulate that the ability of the $\mathrm{NC1}$ domains to bind with high affinities to laminin 5 and collagen IV facilitates stabilization of the structure of the basement membrane itself and that the NC1-collagen I interaction may be less important for stabilization of the dermal-epidermal junction.
\end{abstract}




\section{Introduction}

Structural integrity of skin depends on correct organization of a biological scaffold formed by intercommunicating macromolecules produced by cells of the epidermis and dermis. These two major layers of skin are separated by the basement membrane within which a number of macromolecules bind with each other, thereby contributing to the stability of dermalepidermal junction [1]. To date a number of macromolecules forming the cutaneous basement membrane have been identified and characterized, and among them different laminins and collagen IV are most abundant. Laminin 5, consisting of $\alpha 3 \beta 3 \gamma 2$ chains is most prominent in the total laminin pool while laminin $6(\alpha 3 \beta 1 \gamma 1)$ and laminin $10(\alpha 5 \beta 1 \gamma 1)$ have been identified as minor components of the basement membrane zone $(\mathrm{BMZ})^{1}[2-4]$.

A common characteristic of laminin 5 and collagen IV is their ability to self-assemble into homotypic networks. In addition, through binding and interlacing with other molecules these proteins become a part of complex heterotypic assemblies. For instance, laminin 5 interacts with the basal side of keratinocytes through binding to the $\alpha 6 \beta 4$ and $\alpha 3 \beta 1$ integrins, and it is linked to laminin 6 through a $\beta 3$ chain/ $\alpha 3$ chain cross link [5-7]. An additional link between laminin 5 and keratinocytes is formed by its binding to the extracellular domain of a transmembrane collagen XVII (for review see [8]). The way in which laminin 5 connects with collagen IV within the lamina densa, a layer of the basement membrane facing the papillary dermis, is not fully understood, but it has been proposed that there is no direct connection between these two molecules. Even though laminin 5 does not bind to collagen IV directly it has been postulated that these molecules interact indirectly via the laminin 6/nidogen complex, which binds to collagen IV through interaction with nidogen, a 150-kDa glycoprotein [9-11].

The way in which the basement membrane of stratified squamous epithelia, such as the skin, connects with underlying stroma is not fully understood. It has been demonstrated however, that collagen VII mediates formation of a critical basement membrane/stroma link (for review see $[4,5])$.

Collagen VII consists of a relatively large triple helical domain flanked by the N-terminal $\mathrm{NC} 1$ domain and the C-terminal NC2 domain [12]. Individual collagen VII molecules form antiparallel homodimers stabilized by disulfide bonds formed between the corresponding NC2 domains. After formation of disulfide bonds the collagen VII/collagen VII dimers assemble in a nonstaggered fashion into anchoring fibrils [13-15]. Microscopic analyses combined with 
immunostaining techniques demonstrated that these fibrils form arcade-like structures, which embed themselves with the $\mathrm{NC} 1$ domains of both ends into the lamina densa [16, 17]. It is postulated that the arches of anchoring fibrils interlace with stromal collagen fibrils to form continuous dermal-epidermal architecture $[4,17]$.

Fundamental for collagen VII to function as an anchoring molecule is the ability of its $\mathrm{NC} 1$ domain to bind to other structural molecules of the extracellular matrix. The binding interactions of the $\mathrm{NC} 1$ domain of collagen VII have been studied extensively. It has been demonstrated that the $\mathrm{NC} 1$ domain binds to laminin 5 and collagen IV in a site-specific fashion but results on its ability to interact with collagen I are controversial [18-20]. The binding interactions of the $\mathrm{NC1}$ domain of collagen VII reported to date were analyzed indirectly by employing solid-state assays that require specific antibodies to detect an NC1-bound interactant, but the kinetics of binding and the binding affinities are not known. It has been shown that the laminin $5 / \mathrm{NC} 1$ binding is driven by the interaction between the $\beta 3$ chain of laminin 5 and a fibronectin-like repeat sequence located within the NC1 domain of collagen VII, and that the site-specific $\mathrm{NCl} /$ collagen IV binding is attributed to the binding sites present within a noncollagenous domain of collagen IV (NC1-IV) [20].

The critical role of collagen VII in stabilizing the structure of the BMZ is explicitly demonstrated by the fact that mutations in collagen VII found in patients with dystrophic epidermolysis bullosa, a heritable blistering disease, lead to pathological changes in the structure of the dermal-epidermal junction [21-24]. Moreover, the structure of the BMZ is altered in patients with epidermolysis bullosa acquisita, a disease characterized by the presence of autoantibodies that specifically target the $\mathrm{NC1}$ domain, thereby altering formation of the NC1stabilized structures $[25,26]$.

Here, we studied the mechanisms of the $\mathrm{NC1}$ domain-mediated interaction of collagen VII with laminin 5 and collagen IV. Specifically, employing an optical biosensor we measured directly the binding of the $\mathrm{NC1}$ domain or mini-collagen VII to collagen IV or laminin 5. Based on the binding kinetics we demonstrated that the $\mathrm{NC1}$ domain has a relatively high affinity for laminin 5 and for collagen IV and that, most likely, the NC1 domain utilizes the same site for binding to laminin 5 and collagen IV. Our results therefore suggest that the NC1 domain of collagen VII stabilizes architecture of the basement membrane of skin through its high-affinity binding to laminin 5 and collagen IV. 


\section{Material and methods.}

Proteins for the binding studies - Recombinant mini-procollagen VII (mProVII) consisting of the NC1 domain, a truncated triple-helical region, and the NC2 domain was purified from cell culture media as described [13]. To express recombinant NC1 domain of human collagen VII we employed a DNA fragment encompassing a 1-3870 $\mathrm{bp}^{2}$ region of cDNA encoding human procollagen VII (a kind gift from Dr. Y. Gache, Universite de Nice-Sophia Antipolis, Nice, France). The 1-3870 DNA fragment, which encodes a 1290-amino acid region encompassing the entire $\mathrm{NC1}$ domain and a 37-amino acid fragment of the triple-helical domain, was cloned into a mammalian expression vector (pcDNA3.1, Invitrogen, Inc.), and it was then expressed in HEK 293 (ATCC, CRL-1573) cells, as described [13, 20, 24]. The G418-resistant clones were selected and then, using the NC1-specific antibody, screened for secretion of the $\mathrm{NC1}$ domain into cell culture media, as described [13,24]. Subsequently, the NC1 domain was purified from cell culture media according to the method described for purification of the mProVII [13]. Structural integrity of the purified NC1 domain was analyzed by polyacrylamide gel electrophoresis. Moreover, structural characteristics of rotary-shadowed $\mathrm{NC1}$ domains were evaluated by transmission electron microscopy in the way described for visualization of the mProVII [13].

Monomeric human collagen IV secreted by HT-1080 cells (ATCC, CCL-121) was purified from cell culture media by ion exchange chromatography according to procedures described [27, 28]. Human procollagen I secreted by the human dermal fibroblasts was isolated from cell culture media as described [29]; purified rat laminin 5 was purchased from Chemicon International, Inc.

Biosensor assays of kinetics of the NC1-mediated binding interactions of collagen VII -- To analyze the NC1-mediated binding interactions between collagen VII and laminin 5 or collagen IV we employed the recombinant NC1 domain and the mProVII. Binding assays were carried out using an optical biosensor (Iasys; Affinity Sensors, UK) as described [24]. In brief, to covalently bind the NC1 domain or the mProVII, designated as acceptors, onto the surfaces of sensors, carboxylate groups present on the surface were activated by injection of a 1:1 mixture of $0.1 \mathrm{M}$ N-hydroxysuccinimide and 0.4 M N-Ethyl-3-(3-dimethylaminopropyl) carbodiimide (Pierce). The individual acceptors dissolved in phosphate buffered saline (PBS) were then 
allowed to bind to the activated surface until a response plateau was reached. The residual active groups were blocked by an injection of $100 \mu \mathrm{L}$ of $1 \mathrm{M}$ ethanolamine- $\mathrm{HCl}(\mathrm{pH} 8.5)$. Non-specific binding sites were blocked by the injection of $100 \mu \mathrm{L}$ of a $1 \%$ solution of bovine serum albumin (BSA) dissolved in PBS. Excess BSA was removed by washing the cuvette with PBS containing $0.05 \%$ of Tween-20 (PBST), followed by three consecutive washes with $10 \mathrm{mM} \mathrm{HCl}$. After reequilibration with PBST, the cuvettes were ready for the binding assays. In addition, a control cuvette with immobilized BSA was prepared using the same procedure.

In a series of experiments the following homotypic and heterotypic interactions were analyzed: (i) between the NC1 domain and laminin 5, (ii) between the $\mathrm{NC1}$ domain and collagen IV, and (iii) between the NC1 domain and collagen I. A similar set of binding assays in which the mProVII served as an acceptor instead of the NC1 was also carried out. Moreover, assays of binding between the $\mathrm{NC1}$ domain and the $\mathrm{NC1}$ domain, between the mProVII and the $\mathrm{NC1}$ domain, and between laminin 5 and collagen IV were also performed.

Cuvettes with immobilized acceptors were primed with PBST at $25^{\circ} \mathrm{C}$ for $10 \mathrm{~min}$. A 100 $\mu \mathrm{L}$ sample containing free interactant dissolved in PBST was added to the cuvette, and then the association phase was recorded. Subsequently, the sample was removed, analyte-free PBST was added to the cuvette, and the dissociation phase was recorded. After each assay, the surface of the cuvette was regenerated by washing with $10 \mathrm{mM} \mathrm{HCl}$, followed by equilibration with PBST. During regeneration cycles attention was paid to complete removal of the surface-bound analyte, and the washing continued until a response equal to a baseline value was reached.

For binding assays, free interactants were added at concentrations ranging from $5 \times 10^{-9}$ $\mathrm{M}$ to $1 \times 10^{-8} \mathrm{M}$. Data from the biosensor were analyzed by the global fitting method described by Myszka and Morton [30]. For each assay, the association rate constants $\left(\mathrm{k}_{o n}\right)$ and the dissociation rate constants $\left(\mathrm{k}_{o f f}\right)$ were obtained, and the equilibrium dissociation constants $\left(\mathrm{K}_{d}\right)$ values were calculated from a ratio of $\mathrm{k}_{\text {off }} / \mathrm{k}_{\text {on }}$. Subsequently, the equilibrium association constant $\left(\mathrm{K}_{a}\right)$ values (termed here as the "affinity") were derived as the inverse of $\mathrm{K}_{d}$.

We also analyzed whether NC1-domains bind laminin 5 and collagen IV through the same or different binding sites. In these assays, the NC1 domain was covalently immobilized on a sensor, and then the NC1-coated surface was saturated with collagen IV. After reaching plateau, laminin 5 was added to the cuvette, and measurements of binding events were continued. 


\section{Results}

Proteins for biosensor binding assays - As demonstrated in Fig. 1, the NC1 domain had a predicted molecular mass of $137 \mathrm{kDa}$ [12]. Electrophoresis in nonreducing conditions revealed that some of the NC1 molecules formed homotypic complexes stabilized by the disulfide bonds (Fig. 1) formed between the cysteine residues present within the NC1 domain [12]. Electron microscopy of the recombinant NC1 domains (Fig. 2) confirmed that they had a structure similar to that of the native NC1 domains seen in mProVII. Specifically, the NC1 domains had the length of about $50 \mathrm{~nm}$ and were characterized by the presence of a globular structure on one end, previously determined to represent the $\mathrm{N}$ terminus (Fig. 2) [12].

Electrophoretic separation of procollagen I, laminin 5, collagen IV, and mProVII confirmed their expected structures, which were consistent with previous reports (Fig. 3) [27, 28, $31,32]$. As we reported earlier, collagen IV produced by HT-1080 cells was predominantly composed of the $\alpha 1(\mathrm{IV})$ and $\alpha 2$ (IV) chains $[27,28]$.

\section{The NC1 domain of collagen VII has a relatively high affinity for binding laminin 5 and} collagen IV - In comparison to other techniques used to measure binding between macromolecules, biosensor binding assays are characterized by a number of advantages. In other methods, in general, detecting an interactant bound to an immobilized acceptor is achieved by employing an interactant-specific antibody. In such techniques, however, in order to remove the non-bound fraction of analyzed interactant and the excess of an antibody, extensive washing of the surface on which binding occurs is required prior to final measurements. During such a procedure, dissociation of weakly bound interactant molecules occurs due to the rapid decrease of concentration of a free interactant. Consequently, by utilizing such a method, only the irreversible or relatively strong binding interactions can be detected. In contrast, a biosensor monitors and records, in a real-time fashion, association and dissociation events taking place on a biosensor's surface, so that even weak interaction can be detected.

Based on the biosensor data fitted into a simple bimolecular interaction model (Fig. 4) we calculated the $\mathrm{k}_{\text {on }}$ and $\mathrm{k}_{\text {off }}$ values. These rates were then used to calculate the $\mathrm{K}_{d}$ and $\mathrm{K}_{a}$ values (Table 1). Analyses of binding of the $\mathrm{NC1}$ domain (Fig. 4, Table 1) demonstrated its affinity to laminin 5 and collagen IV with $\mathrm{K}_{a}$ values of $4.8 \times 10^{8} \mathrm{M}^{-1}$ and $3.6 \times 10^{8} \mathrm{M}^{-1}$, respectively. In 
contrast, the $\mathrm{K}_{a}$ value of $1.6 \times 10^{6} \mathrm{M}^{-1}$ for binding of collagen I indicates relatively low affinity of the $\mathrm{NC1}$ domain for collagen I binding. Because of the decrease in the signal detected by the biosensor, indicating dissociation of complexes, the $\mathrm{NC1}$ domain-mediated binding between collagen VII and laminin 5 or collagen IV is considered a reversible process (Fig. 4). In the same experimental conditions there was no detectable $\mathrm{NC} 1 / \mathrm{NC} 1$ or $\mathrm{NC} 1 / \mathrm{mPro} \mathrm{VII}$ binding (data not shown), thereby indicating low affinity between individual $\mathrm{NCl}$ domains. There was no detectable binding of laminin 5 to collagen IV (Table 1).

Binding interactions of the individual $\mathrm{NC} 1$ domains were corroborated by the binding assays of the mProVII (data not shown), which in addition to the $\mathrm{NC1}$ domain includes a truncated triple-helical region and the NC2 domain [13, 24]. The affinities of the mProVII for laminin 5, collagen IV and collagen I were similar to the affinities of the $\mathrm{NC} 1$ domain for those proteins (Table 1). There were, however, differences between binding kinetics recorded for $\mathrm{mProVII} /$ laminin 5 and $\mathrm{NCl} /$ laminin 5 binding (Table 1).

The NC1-domain utilizes the same or closely located sites for binding of laminin 5 and collagen IV -- As indicated in Fig. 5, after saturating the surface-immobilized NC1 domains with collagen IV, there was no additional binding of laminin 5 to the $\mathrm{NCl} /$ collagen IV complex. Because as we established earlier, there was no detectable binding between laminin 5 and the NC1 domain (Table 1), such lack of the additional binding to the collagen IV-saturated NC1 domain could not be attributed to trapping of free laminin 5 by free collagen IV molecules present in solution. These data suggest that the binding of the $\mathrm{NC1}$ domain to laminin 5 and collagen IV is mediated through either the same or a closely located binding site. To confirm the specificity of analyzed interactions, we examined the ability of BSA to bind to the NC1 and mProVII. The injection of BSA (not shown) onto surface-immobilized NC1 domain and mProVII did not exhibit the typical association or dissociation curves that were observed with laminin 5, collagen IV and collagen I. 


\section{Discussion}

Noncovalent interactions between macromolecules are essential for the formation of supramolecular structures of biological scaffolds that shape the architecture of tissues and organs. A macromolecular scaffold of skin is a complex structure formed by a number of molecules interacting with each other in a site-specific manner. One of the distinct morphological features of skin is that it is composed of two principal layers, namely the epidermis and dermis, separated by the basement membrane, a structure that serves as an attachment zone on which structural macromolecules produced by epidermal and dermal cells interact, thereby contributing to the formation of the complex dermal-epidermal junction [33].

Collagen VII takes part in stabilizing the dermal-epidermal junction and this protein forms anchoring fibrils whose function is to provide a link between the basement membrane and stroma [1, 18]. It has been established that the NC1-mediated binding of anchoring fibrils to elements present within basement membrane and interlacing of those fibrils with stromal collagen fibrils composed predominantly of collagen I facilitates this link (Fig 6) [4].

We studied the role of the NC1 domains of collagen VII in stabilizing the base membrane through their interaction with laminin 5 and collagen IV. The recombinant NC1 domain we employed in our studies was present in the form of a monomer as well as covalently cross-linked homodimers but, as previously demonstrated by Chen et al., [19] the recombinant NC1 domain interacts specifically with its binding partners regardless of its monomeric, dimeric or trimeric form.

We demonstrated that the affinities of the $\mathrm{NC} 1$ domain for binding to laminin 5 and collagen IV are relatively high. Because of the lower $\mathrm{k}_{o n}$, a smaller affinity of the mProVII for laminin 5 in comparison to that of the $\mathrm{NC1}$ domain for laminin 5 could be result of a steric hindrance imposed by the presence of the triple-helical region and the NC2 domain present in the mProVII.

In the past, predictions have been made that the anchoring fibrils could form extended structures, so that the $\mathrm{NC1}$ domains on end could be embedded into the lamina densa while the $\mathrm{NC1}$ domains from the opposite end could be embedded in anchoring plaques, basement membrane-like structures within the dermis [34]. These anchoring plaques however, were later found to be a microscopic artifact (for review see [4]). More recently, evidence for the existence 
of arch-like anchoring fibrils, rather than extended ones, embedded with both $\mathrm{N}$ termini into lamina densa was presented (Fig. 6). Therefore, the relatively low affinities of the NC1 domain and mProVII for collagen I, as reported here, suggest that it is unlikely that the NC1 domains of collagen VII molecules play any significant role in connecting stromal collagen fibrils with the lamina densa through direct $\mathrm{NC1} /$ collagen I binding. To explain how anchoring fibrils interact with underlying stroma $[16,17]$ it has been proposed that some of the stromal collagen fibrils approach the lamina densa and interlace with arcade-like structures of anchoring fibrils (Fig. 6) (for review see [4]). Such interlacing and the relatively low affinity of the NC1 for binding collagen I observed in our studies indicate that the role of collagen VII in stabilizing the dermalepidermal junction through its interaction with collagen fibrils has a "passive" character. In contrast, the higher affinity of the $\mathrm{NC1}$ domain of collagen VII for binding of laminin 5 and collagen IV indicates its "active" role in stabilizing the basement membrane-dermal association. Measurements of the binding of the NC1 domain to collagen I presented here support previous reports by Chen et al. [20] who showed the NC1/collagen I interaction in solid-state binding assays in which an anti-NC1 antibody was used to detect the $\mathrm{NC1}$ /collagen I complexes.

Our binding data also shed light on the mechanism of assembly of anchoring fibrils. The lack of detectable $\mathrm{NC1} / \mathrm{NC1}$ interaction described here and a relatively high $\mathrm{mProVII} / \mathrm{mPro}$ VII affinity on the order of $10^{8} \mathrm{M}^{-1}$ reported earlier [24], indicate that the lateral assembly of collagen VII/collagen VII dimers during formation of anchoring fibrils is not likely to be driven by the $\mathrm{NC1} / \mathrm{NC} 1$ interaction but rather by interactions between other domains..

Employing biosensor binding assays we also demonstrated that the collagen IV binding site within the $\mathrm{NC} 1$ domain is the same or is positioned closely to that for binding laminin 5 . In previous reports the laminin 5-binding site in the $\mathrm{NC1}$ domain of collagen VII was mapped to be located within the fibronectin III-like repeats [19]. Consequently, we propose that it is likely that the $\mathrm{NC1}$ /collagen IV binding could involve the same domain. A similar multifunctionality of the binding domain was identified in cartilage oligomeric matrix protein by Holden et al. [35] who showed that this protein utilizes the same site for binding to collagen II and collagen IX.

The values for binding affinities we calculated help to understand the molecular bases of the stability of the dermal-epidermal junction by pointing to the critical role of the $\mathrm{NCl}$ domain of collagen VII in mediating intermolecular interactions between major structural proteins of the basement membrane zone. It is expected that the binding characteristics of the NC1 domain 
could change not only as a result of mutations in collagen VII found in patients with dystrophic epidermolysis bullosa but also in autoimmune diseases, such as epidermolysis bullosa acquisita, caused by antibodies against the $\mathrm{NC} 1$ domain. Since the most studied antibodies have relatively high affinities for their respective antigens, frequently in the range of $10^{8}-10^{10} \mathrm{M}^{-1}$, and because the interaction of the $\mathrm{NC1}$ domain with collagen IV and laminin 5 is reversible (see Fig. 4), the anti-NC1 antibodies could alter the binding of the NC1 domain to laminin 5 and collagen IV, thereby causing damage to the structure of the basement membrane zone. Since the affinity of the $\mathrm{NC1}$ domain for laminin 5 is lower than that for collagen IV, the presence of the anti-NC1 antibodies would be more devastating for the formation of the $\mathrm{NC1} /$ laminin 5 complexes.

In summary, our results on the NC1domain-mediated interactions of collagen VII with laminin 5, collagen IV, and collagen I provide molecular bases for understanding the role of this domain in stabilizing the dermal-epidermal junction. 


\section{Acknowledgements}

We thank Dr. Yannick Gache for a DNA constructs encoding the NC1 domain, Dr. David E. Birk and Biao Zuo for expert technical assistance with the electron microscopy. This study was supported by the NIH/NIAMS grant P01-AR38923. 


\section{References}

[1] R. E. Burgeson, A. M. Christiano, The dermal-epidermal junction, Curr Opin Cell Biol. 9 (1997) 651-658.

[2] M. Aumailley, P. Rousselle, Laminins of the dermo-epidermal junction, Matrix Biol. 18 (1999) 19-28.

[3] C. M. Niessen, E. H. Hulsman, E. S. Rots, P. Sanchez-Aparicio, A. Sonnenberg, Integrin alpha 6 beta 4 forms a complex with the cytoskeletal protein HD1 and induces its redistribution in transfected COS-7 cells, Mol Biol Cell. 8 (1997) 555-566.

[4] E. Adachi, I. Hopkinson, T. Hayashi, Basement-membrane stromal relationships: interactions between collagen fibrils and the lamina densa, Int Rev Cytol. 173 (1997) 73-156.

[5] R. F. Ghohestani, K. Li, P. Rousselle, J. Uitto, Molecular organization of the cutaneous basement membrane zone, Clin Dermatol. 19 (2001) 551-562.

[6] M. F. Champliaud, G. P. Lunstrum, P. Rousselle, T. Nishiyama, D. R. Keene, R. E. Burgeson, Human amnion contains a novel laminin variant, laminin 7, which like laminin 6, covalently associates with laminin 5 to promote stable epithelial-stromal attachment, J Cell Biol. 132 (1996) 1189-1198.

[7] W. G. Carter, M. C. Ryan, P. J. Gahr, Epiligrin, a new cell adhesion ligand for integrin alpha 3 beta 1 in epithelial basement membranes, Cell. 65 (1991) 599-610.

[8] J. Uitto, L. Pulkkinen, Molecular complexity of the cutaneous basement membrane zone, Mol Biol Rep. 23 (1996) 35-46.

[9] J. W. Fox, U. Mayer, R. Nischt, M. Aumailley, D. Reinhardt, H. Wiedemann, K. Mann, R. Timpl, T. Krieg, J. Engel, et al., Recombinant nidogen consists of three globular domains and mediates binding of laminin to collagen type IV, Embo J. 10 (1991) 3137-3146.

[10] M. Aumailley, C. Battaglia, U. Mayer, D. Reinhardt, R. Nischt, R. Timpl, J. W. Fox, Nidogen mediates the formation of ternary complexes of basement membrane components, Kidney Int. 43 (1993) 7-12.

[11] U. Mayer, R. Nischt, E. Poschl, K. Mann, K. Fukuda, M. Gerl, Y. Yamada, R. Timpl, A single EGF-like motif of laminin is responsible for high affinity nidogen binding, Embo J. 12 (1993) 1879-1885. 
[12] A. M. Christiano, D. S. Greenspan, S. Lee, J. Uitto, Cloning of human type VII collagen. Complete primary sequence of the alpha 1 (VII) chain and identification of intragenic polymorphisms, J Biol Chem. 269 (1994) 20256-20262.

[13] M. Colombo, R. J. Brittingham, J. F. Klement, I. Majsterek, D. E. Birk, J. Uitto, A. Fertala, Procollagen VII self-assembly depends on site-specific interactions and is promoted by cleavage of the NC2 domain with procollagen C-proteinase, Biochemistry. 42 (2003) 11434-11442.

[14] A. Rattenholl, W. N. Pappano, M. Koch, D. R. Keene, K. E. Kadler, T. Sasaki, R. Timpl, R. E. Burgeson, D. S. Greenspan, L. Bruckner-Tuderman, Proteinases of the bone morphogenetic protein-1 family convert procollagen VII to mature anchoring fibril collagen, J Biol Chem. 277 (2002) 26372-26378.

[15] L. Bruckner-Tuderman, O. Nilssen, D. R. Zimmermann, M. T. Dours-Zimmermann, D. U. Kalinke, T. Gedde-Dahl, Jr., J. O. Winberg, Immunohistochemical and mutation analyses demonstrate that procollagen VII is processed to collagen VII through removal of the NC-2 domain, J Cell Biol. 131 (1995) 551-559.

[16] J. R. McMillan, M. Akiyama, H. Shimizu, Ultrastructural orientation of laminin 5 in the epidermal basement membrane: an updated model for basement membrane organization, $\mathrm{J}$ Histochem Cytochem. 51 (2003) 1299-1306.

[17] H. Shimizu, A. Ishiko, T. Masunaga, Y. Kurihara, M. Sato, L. Bruckner-Tuderman, T. Nishikawa, Most anchoring fibrils in human skin originate and terminate in the lamina densa, Lab Invest. 76 (1997) 753-763.

[18] R. E. Burgeson, G. P. Lunstrum, B. Rokosova, C. S. Rimberg, L. M. Rosenbaum, D. R. Keene, The structure and function of type VII collagen, Ann N Y Acad Sci. 580 (1990) 32-43.

[19] M. Chen, M. P. Marinkovich, J. C. Jones, E. A. O'Toole, Y. Y. Li, D. T. Woodley, NC1 domain of type VII collagen binds to the beta3 chain of laminin 5 via a unique subdomain within the fibronectin-like repeats, J Invest Dermatol. 112 (1999) 177-183.

[20] M. Chen, M. P. Marinkovich, A. Veis, X. Cai, C. N. Rao, E. A. O'Toole, D. T. Woodley, Interactions of the amino-terminal noncollagenous (NC1) domain of type VII collagen with extracellular matrix components. A potential role in epidermal-dermal adherence in human skin, J Biol Chem. 272 (1997) 14516-14522.

[21] J. Uitto, A. M. Christiano, Dystrophic forms of epidermolysis bullosa, Semin Dermatol. 12 (1993) 191-201. 
[22] J. Uitto, L. Pulkkinen, Molecular genetics of heritable blistering disorders, Arch Dermatol. 137 (2001) 1458-1461.

[23] L. Pulkkinen, F. Ringpfeil, J. Uitto, Progress in heritable skin diseases: molecular bases and clinical implications, J Am Acad Dermatol. 47 (2002) 91-104.

[24] R. Brittingham, M. Colombo, H. Ito, A. Steplewski, D. E. Birk, J. Uitto, A. Fertala, Single Amino Acid Substitutions in Procollagen VII Affect Early Stages of Assembly of Anchoring Fibrils, J Biol Chem. 280 (2005) 191-198.

[25] D. T. Woodley, C. Chang, P. Saadat, R. Ram, Z. Liu, M. Chen, Evidence that anti-type VII collagen antibodies are pathogenic and responsible for the clinical, histological, and immunological features of epidermolysis bullosa acquisita, J Invest Dermatol. 124 (2005) 958964.

[26] J. C. Lapiere, D. T. Woodley, M. G. Parente, T. Iwasaki, K. C. Wynn, A. M. Christiano, J. Uitto, Epitope mapping of type VII collagen. Identification of discrete peptide sequences recognized by sera from patients with acquired epidermolysis bullosa, J Clin Invest. 92 (1993) 1831-1839.

[27] A. Fertala, A. L. Sieron, A. Ganguly, S. W. Li, L. Ala-Kokko, K. R. Anumula, D. J. Prockop, Synthesis of recombinant human procollagen II in a stably transfected tumour cell line (HT1080), Biochem J. 298 (1994) 31-37.

[28] D. G. Kisiel, J. J. Calvete, J. Katzhendler, A. Fertala, P. Lazarovici, C. Marcinkiewicz, Structural determinants of the selectivity of KTS-disintegrins for the alphalbetal integrin, FEBS Lett. 577 (2004) 478-482.

[29] A. Torre-Blanco, E. Adachi, A. M. Romanic, D. J. Prockop, Copolymerization of normal type I collagen with three mutated type I collagens containing substitutions of cysteine at different glycine positions in the alpha 1 (I) chain, J Biol Chem. 267 (1992) 4968-4973.

[30] D. G. Myszka, T. A. Morton, CLAMP: a biosensor kinetic data analysis program, Trends Biochem Sci. 23 (1998) 149-150.

[31] G. Giannelli, J. Falk-Marzillier, O. Schiraldi, W. G. Stetler-Stevenson, V. Quaranta, Induction of cell migration by matrix metalloprotease-2 cleavage of laminin-5, Science. 277 (1997) 225-228.

[32] B. E. Vogel, R. Doelz, K. E. Kadler, Y. Hojima, J. Engel, D. J. Prockop, A substitution of cysteine for glycine 748 of the alpha 1 chain produces a kink at this site in the procollagen I 
molecule and an altered N-proteinase cleavage site over $225 \mathrm{~nm}$ away, J Biol Chem. 263 (1988) 19249-19255.

[33] R. A. Briggaman, C. E. Wheeler, Jr., The epidermal-dermal junction, J Invest Dermatol. 65 (1975) 71-84.

[34] D. R. Keene, L. Y. Sakai, G. P. Lunstrum, N. P. Morris, R. E. Burgeson, Type VII collagen forms an extended network of anchoring fibrils, J Cell Biol. 104 (1987) 611-621.

[35] P. Holden, R. S. Meadows, K. L. Chapman, M. E. Grant, K. E. Kadler, M. D. Briggs, Cartilage oligomeric matrix protein interacts with type IX collagen, and disruptions to these interactions identify a pathogenetic mechanism in a bone dysplasia family, J Biol Chem. 276 (2001) 6046-6055.

[36] M. Aumailley, L. Bruckner-Tuderman, W. G. Carter, R. Deutzmann, D. Edgar, P. Ekblom, J. Engel, E. Engvall, E. Hohenester, J. C. Jones, H. K. Kleinman, M. P. Marinkovich, G. R. Martin, U. Mayer, G. Meneguzzi, J. H. Miner, K. Miyazaki, M. Patarroyo, M. Paulsson, V. Quaranta, J. R. Sanes, T. Sasaki, K. Sekiguchi, L. M. Sorokin, J. F. Talts, K. Tryggvason, J. Uitto, I. Virtanen, K. von der Mark, U. M. Wewer, Y. Yamada, P. D. Yurchenco, A simplified laminin nomenclature, Matrix Biol. 24 (2005) 326-332. 


\section{Figure legends}

\section{Figure 1.}

Electrophoretic analysis of purified recombinant NC1 domain of collagen VII. The NC1 domain expressed in HEK 293 cells was analyzed in reducing $(+\beta)$ and nonreducing $(-\beta)$ conditions. Electrophoretic migration of the $\mathrm{NC1}$ domain in reducing conditions was consistent with its predicted molecular mass of $137 \mathrm{kDa}$, while migration in nonreducing conditions suggests formation of homotypic dimers. Symbols: NC1m, the NC1 domain in its monomeric form; $\mathrm{NC1} / \mathrm{NC1}$, a dimer formed by the $\mathrm{NC1}$ domains linked by the disulfide bonds; $+\beta$ and $-\beta$ indicate the presence $(100 \mu \mathrm{M})$ or absence of a reducing agent, respectively. Molecular mass markers are indicated in the left lane.

\section{Figure 2.}

Visualization of the rotary shadowed $\mathrm{NC1}$ domain and the mProVII by transmission electron microscopy. Upper panel: some of the NC1 domains seem to be connected at one end, most likely due to formation of a disulfide bond (see Fig. 1). Lower panel: mProVII molecule is characterized by the presence of the $\mathrm{NC} 1$ domain, a truncated triple-helical region, and the $\mathrm{NC} 2$ domain. Symbols: NC1 and NC2, the N-terminal and C-terminal noncollagenous domains of collagen VII, respectively; TH, truncated triple-helical region of procollagen VII [13]. Bar $=100$ nm.

\section{Figure 3.}

Electrophoretic analysis of proteins used in the biosensor binding assays (see Fig. 4). Electrophoretic separation of procollagen I (P-I), laminin 5 (L-5), collagen IV (C-IV), and mProVII (C-VII) confirms their correct overall structure. Symbols: Pro- $\alpha 1(\mathrm{I})$ and Pro- $\alpha 2(\mathrm{I})$, pro$\alpha 1$ and pro- $\alpha 2$ chains of procollagen I; $\alpha 3, \beta 3$, and $\gamma 2$, chains of laminin 5 ; $\alpha(\mathrm{IV})$, co-migrating $\alpha 1$ and $\alpha 2$ chains of collagen IV; mPro- $\alpha 1$ (VII), $\alpha 1$ chain of mini procollagen VII. Molecular mass markers are indicated in the left lane. 


\section{Figure 4.}

Association and dissociation curves illustrating kinetics of the heterotypic binding between NC1 domains and collagen IV (C-IV), laminin 5 (L-5) or collagen I (P-I). In each panel, the curves represent association and dissociation events during the heterotypic interaction between immobilized $\mathrm{NC} 1$ domain and free interactants present at concentrations ranging from $5 \times 10^{-9} \mathrm{M}$ to $1 \times 10^{-8} \mathrm{M}$. Lower panels are a corresponding graphic presentation of the residuals of curves fitted to the data points collected by a biosensor.

\section{Figure 5.}

Biosensor analysis of collagen IV and laminin 5 binding sites in the NC1 domains. The recombinant NC1 domain was immobilized on a surface of a biosensor. Subsequently, the NC1coated surface was saturated by collagen IV (C-IV) followed by adding laminin 5 (L-5). Lack of additional response from the sensor after adding laminin 5 indicates that the collagen IVsaturated NC1 domain was not able to bind laminin 5, thereby suggesting that the laminin 5binding site corresponds to or is closely located to that for binding of collagen IV. Upon washing the surface with a free solvent the $\mathrm{NC} 1 /$ collagen IV complex dissociated, thereby confirming a reversible nature of the $\mathrm{NC1}$ /collagen IV binding. Arrows indicate time points at which $\mathrm{C}$-IV, L5 , and free solvent wash were added.

\section{Figure 6.}

Schematic representation of the dermal-epidermal junction depicting macromolecules whose binding interactions were analyzed. The cartoon at left depicts basal keratinocytes $(\mathrm{K})$ interacting through binding to laminin 5 (L-5) and laminin 6 (L-6) to collagen IV (C-IV) network. Moreover,

anchoring fibrils consisting of collagen VII (C-VII) that bind to collagen IV and laminin 5 and interlace with collagen I fibrils formed in the dermis by fibroblasts (F) are presented. At right, a detail of a region of interaction between the $\mathrm{NC1}$ domain of collagen VII (NC1-VII) and the 
$\mathrm{NC} 1$ domain of collagen IV (NC1-IV), and a short arm of the $\beta 3$ chain of laminin 5 ( $\beta 3$ ) is presented. Laminin 6 and nidogen are also present in the schematics. 


\section{Footnotes}

${ }^{1}$ A modified nomenclature for laminins has been recently proposed, in which the laminins are identified on the basis of their $\alpha, \beta$, and $\gamma$ subunits [36]. Thus laminin 5 is termed as laminin 332, laminin 6 as laminin 311, and laminin 10 as laminin 511.

${ }^{2}$ The numbers indicate the first base of the translation initiation codon. 


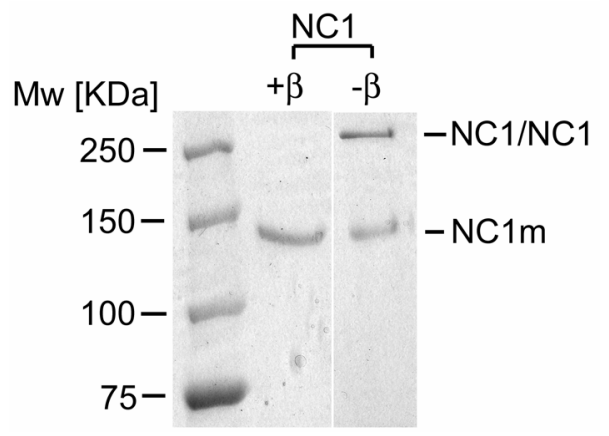

Figure 1. 


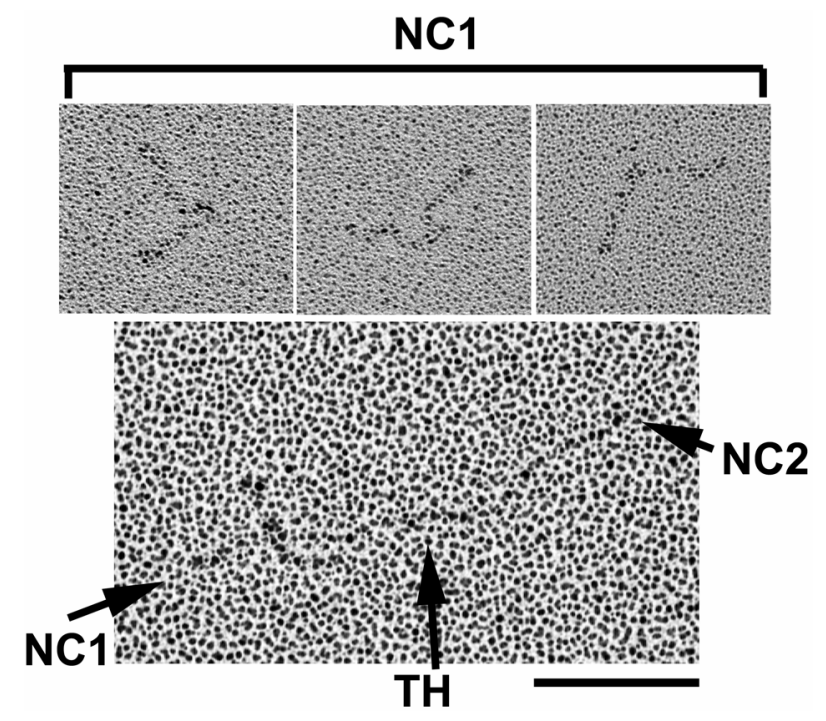

Figure 2. 


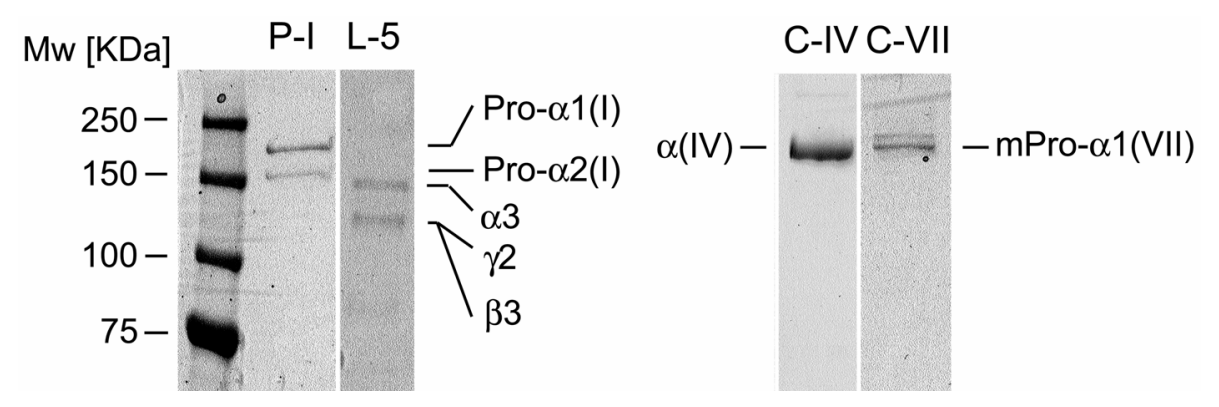

Figure 3. 

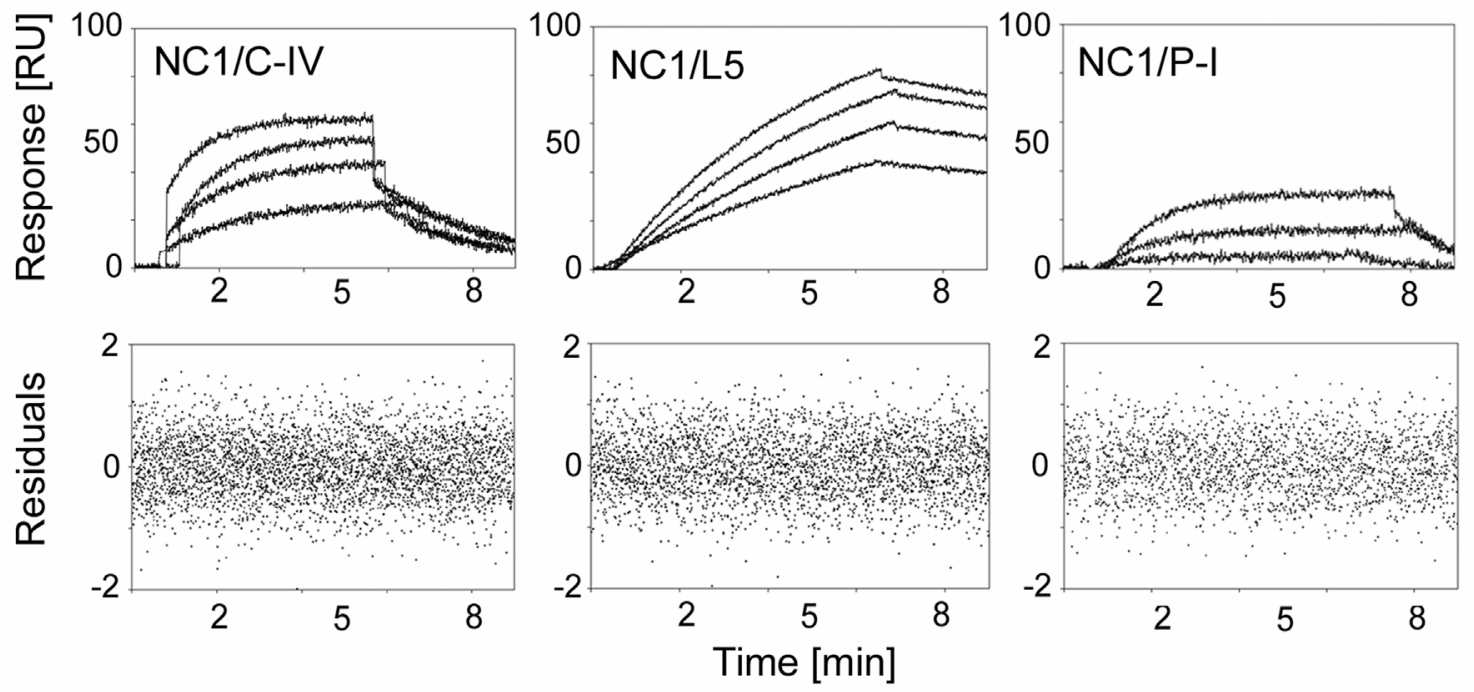

Figure 4. 


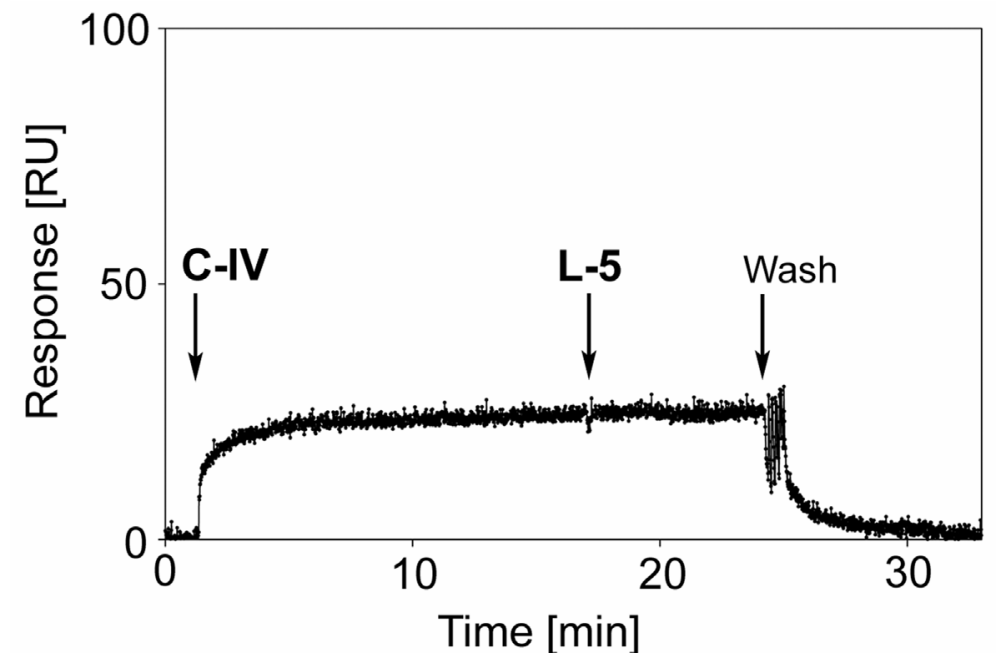

Figure 5. 


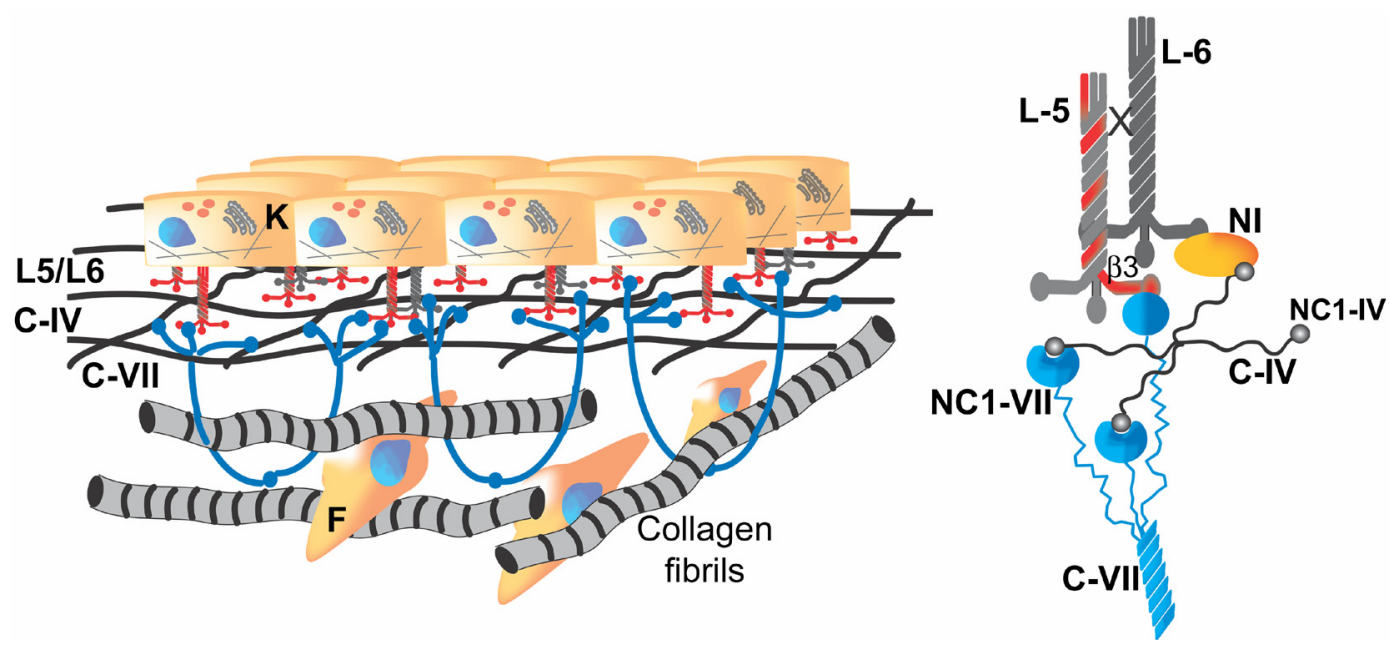

Figure 6. 